NASA

Technical

Paper

3023

1990

\section{Mode-Medium Instability and Its Correction With a Gaussian Reflectivity Mirror}

K. L. Webster

George C. Marshall Space Flight Center

Marshall Space Flight Center, Alabama

C. C. Sung

The University of Alabama in Huntsville

Huntsville, Alabama 



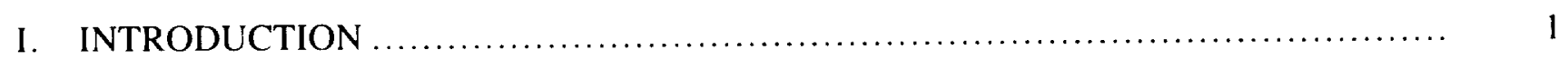

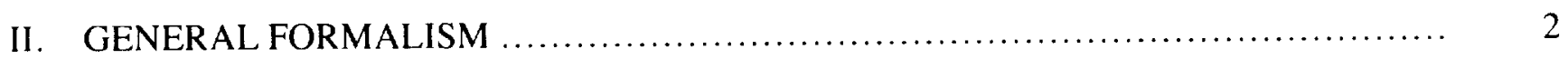

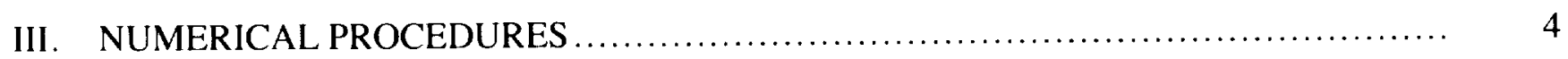

A. Free-Space Propagation ....................................................... 4

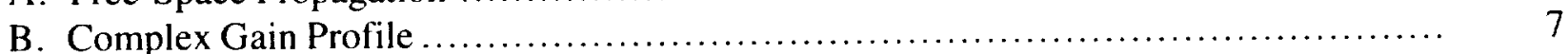

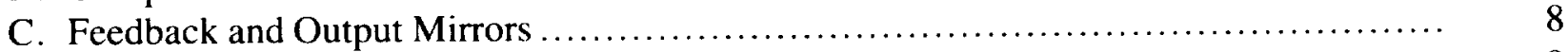

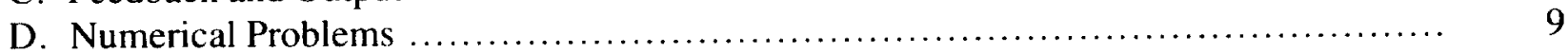

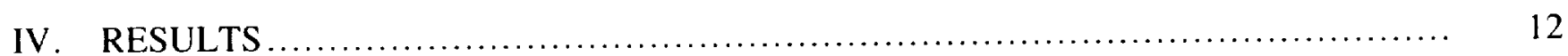

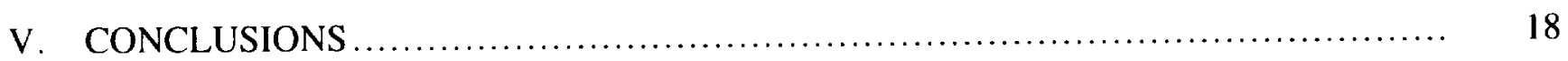

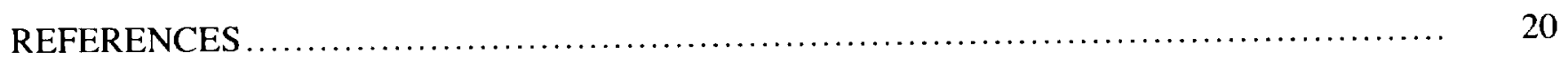




\section{LIST OF ILLUSTRATIONS}

Figure

Title

Page

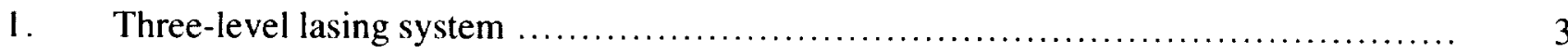

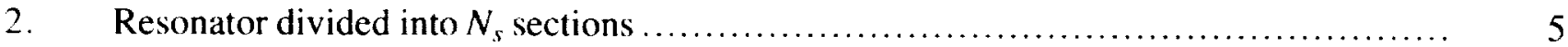

3. Variations of the Hanning filter: (a) normal and (b) truncated.

The maximum frequency of the FFT is proportional to the number

of data points

4. Time evolution of intensity and phase profile just before the hard-edge output mirror with $N_{e q}=2.0$ and $P=0.3 \mathrm{~atm}$

5. Hard-edge resonator beam quality as a function of time with

$P=0.3 \mathrm{~atm}$ and $N_{e q}=$ (a) 1.0 , (b) 2.0 , (c) 3.0 , (d) 4.0 ,

and (e) 5.0

6. Peak value of the $G_{i}$ profile just before the hard-edge output mirror and as a function of time with $P=0.3$ atm and $N_{e q}=$ (a) 1.0 , (b) 2.0 ,

(c) 3.0 , (d) 4.0 , and (e) $5.0 . G_{i}$ is in units of radians

7. Hard-edge resonator beam quality as a function of time with $N_{e q}=2.0$

and $P=$ (a) 0.3 , (b) 0.5 , (c) 0.7 , and (d) $0.9 \mathrm{~atm}$

8. Peak value of the $G_{i}$ profile just before the hard-edge output mirror and as a function of time with $N_{e q}=2.0$ and $P=$ (a) 0.3 , (b) 0.5 , (c) 0.7 , and (d) $0.9 \mathrm{~atm} . G_{i}$ is in units of radians

9. Time evolution of intensity and phase profile just before the soft-edge output mirror with $N_{e q}=2.0$ and $P=0.3 \mathrm{~atm}$

10. Soft-edge resonator beam quality as a function of time with $P=0.3 \mathrm{~atm}$ and $N_{e q}=$ (a) 1.0 , (b) 2.0, (c) 3.0, and (d) 4.0

11. Peak value of the $G_{i}$ profile just before the soft-edge output mirror and as a function of time with $P=0.3$ atm and $N_{e q}=$ (a) 1.0 , (b) 2.0 , (c) 3.0 , and (d) $4.0 . G_{i}$ is in units of radians 
TECHNICAL PAPER

\section{MODE-MEDIUM INSTABILITY AND ITS CORRECTION WITH
A GAUSSIAN REFLECTIVITY MIRROR}

\section{INTRODUCTION}

The mode-medium instability (MMI) effect is due to the vibrational-to-translational relaxation of the upper and lower $\mathrm{CO}_{2}$ lasing levels which results in an intensity-dependent heating rate that is greatest where the resonator intensity is highest. The uneven heating rate in an unstable laser resonator with hardedge output mirror is attributed mainly, although not wholly, to the radiation intensity variations produced by diffraction from the edge of the output mirror. The uneven heating rate leads to acoustic density perturbations and, thus, spatial and temporal variations in the dielectric constant which in turn causes the radiation to be diffracted even more. The change in intensity causes more variations in the heating rate and the feedback cycle is complete. As a result, the quality of the output beam degrades with time, and for pulses of sufficient intensity and duration the beam quality can be completely destroyed. Thus, MMI is of major importance in designing high-power long-pulse $\mathrm{CO}_{2}$ lasers. A review of the earlier experimental work is given in References 1 through 8 .

The numerical modeling of MMI involves the solution of two coupled nonlinear partial differential equations as shown later. Recently, the asymptotic expansion [9] of Fox and Li's equation [10] was used to study the qualitative time evolution of the laser beam in the unstable resonator. The computation, however, is only valid for very large Fresnel numbers. As expected, larger Fresnel number and smaller gas pressure were found to give higher beam quality for a longer period of time. Furthermore, the deterioration of the laser beam is initiated by the magnitude of the diffraction ripple at the early stage of lasing. Although the quantitative results are new, the physical mechanisms have been known for a long time and have prompted many suggestions for improvement.

The suggestions for improving beam quality include a stepped mirror $[11,12]$, soft-edge mirror [6], pressure reduction [6], and phase conjugation [7]. The stepped and soft-edge mirrors are alternate mirror designs aimed at reducing or eliminating the diffraction ripples produced by the edge of the output mirror of the unstable resonator. The stepped mirror is characterized by a step phase discontinuity which produces an additional set of edge waves that are designed to interfere with and cancel the other set of edge waves produced by the outer edge of the mirror. On the other hand, the soft-edge mirror is characterized by a tapered edge reflectivity which eliminates the diffraction produced by the hard-edge output mirror. The Gaussian reflectivity mirror [13-17] is the most popular soft-edge mirror due to its ability to transform the diffraction modulated modes of the unstable resonator into a smooth Gaussian mode without sacrificing the large mode volume and parallel output beam of the unstable resonator. A quantitative investigation, including MMI, however, is not available. Another. This type of possible solution would be very difficult to implement since the beam quality. It has been second scales. Pressure reduction is also an important me percentage of near-field energy delivered to demonstrated [6] that a reduction in pressure increases the percentage of near-field output power. For higher
the far-field. However, as will be shown later, this is at the expense of total out 
power it is still necessary to operate at higher pressure. It has also been suggested that phase conjugation 171, due to its ability to cancel medium induced phase distortions, may be able to reduce the MMI effect. Our motivation is to give a quantitative study of MMI for small Fresnel number to complement Reference 9 and to study the corrective mechanisms suggested previously by others.

In order to supplement the work in Reference 9 for large Fresnel numbers, this paper investigates the qualitative properties for small Fresnel numbers, $\leqslant 5$, and the quantitative advantages of using the Gaussian reflectivity mirror for improving the beam quality as a function of time. The presence of a somewhat complicated mirror and an active nonlinear medium causes a great deal of numerical difficulty. The gas density equation and Maxwell's equations are solved in one transverse dimension along with several gain sheets in the longitudinal dimension in constrast with early works [18] which employ only one sheet. The use of additional gain sheets improves the quantitative aspects of the results. Although there are previous works [13-17] which have addressed the use of Gaussian reflectivity mirrors in unstable resonators, none have numerically investigated their use in correcting the mode instability arising from the MMI. This paper reports the first time resolved mode calculations in an unstable resonator with a Gaussian reflectivity mirror and with $\mathrm{MMI}$ effects included in the calculation.

In the following sections, the standard formalism, for both the hard-edge and Gaussianreflectivity resonators, is first presented. The numerical procedure is described in the third section with explanations of some subtleties of the computations. The results on the time evolution of the beam quality and peak power output are presented in the fourth section. A discussion concluding the paper is
given in the end.

\section{GENERAL FORMALISM}

In the active medium, the dielectric constant can be written as [9]

$$
\epsilon=1+\Delta \epsilon=1+4 \pi a \frac{\rho}{m_{0}}+i g \frac{c}{\omega},
$$

where $\Delta \epsilon$ is a spatial and time-dependent complex number, $\alpha$ is the molecular polarizability, $m_{0}$ is the molecular mass, $c$ is the speed of light, and $\omega$ is the frequency. In the three-level $\mathrm{CO}_{2}$ lasing model, as proportional the $1, \rho$ is proportional to the $\mathrm{CO}_{2}$ in the ground level, whereas $g$, the gain constant, is proportional to the density in the highest level [19]. Specifically, we write

$$
g=\frac{g_{0}}{1+\frac{I}{I_{\text {sat }}}},
$$

where $g_{0}$ is the small signal gain and $I_{\text {sar }}$ is the saturation intensity. An equation of state for $\rho$ has been
derived earlier [4], 


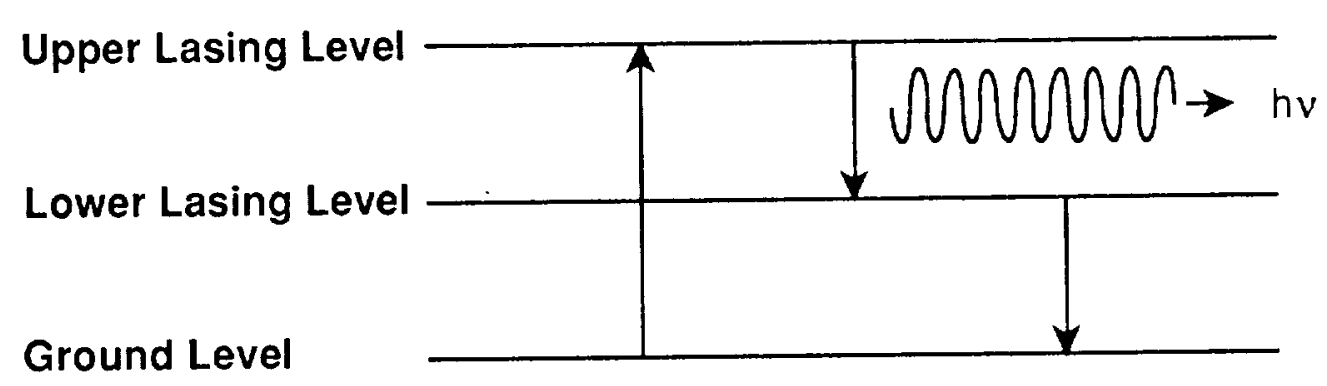

Figure 1. Three-level lasing system.

$$
\frac{\partial}{\partial t}\left(\frac{\partial^{2}}{\partial t^{2}}-C_{s}^{2} \nabla^{2} Q\right)=(\gamma-1) \nabla^{2} q
$$

where $C_{s}$ is the speed of sound, $\gamma=1.7$ is the ratio of specific heat at constant pressure to the specific heat at constant volume, and $q$ is the heating rate per unit volume which is related to the intensity $I$ by [9]

$$
q=\left(\frac{v_{1}}{v}\right) g I+\left(\frac{v_{2}}{v}\right) g J_{s a t}=g_{0} J_{s a t}\left(\frac{v_{2}}{v}\right)\left(\frac{1+\frac{v_{1}}{v_{2}}|u|^{2}}{1+|u|^{2}}\right)
$$

where equation (2) has been included and $/$ has been normalized according to

$$
|u|^{2}=\frac{I}{I_{s a t}}
$$

The solution of equation (3) is [4]

$$
Q(x, t)=\frac{-(\gamma-1)}{C_{s}^{2}} \times \int_{0}^{t} d t^{\prime}\left[q\left(x, t^{\prime}\right)-\frac{1}{2} q\left(x+C_{s}\left(t-t^{\prime}\right), t^{\prime}\right)-\frac{1}{2} q\left(x-C_{s}\left(t-t^{\prime}\right), t^{\prime}\right)\right] .
$$

Equations (1) through (6) are to be solved with the Maxwell equation for the field amplitude,

$$
E=\tilde{E} \exp [i(\omega t-k z)],
$$

where $\bar{E}$ satisfies the envelope equation, 


$$
\left[\frac{\partial^{2}}{\partial x^{2}}-2 i k \frac{\partial}{\partial z}+2 i \frac{\omega}{c^{2}} \frac{\partial}{\partial t}\right] \bar{E}=-k^{2} \Delta \epsilon \bar{E}
$$

The wave number $k$ and the frequency $\omega$ are related by $\omega=c k$, and

$$
l=\frac{1}{2} n \cdot|\tilde{E}|^{2}
$$

The set of equations given in (1) to (9) would be a satisfactory model for the MMI. In actual execution of the solution, the following approximations and limitations are introduced: (i) The model is limited to one transverse dimension which does not change the qualitative significance of the results. (ii) The Fast Fourier Transform with 256 data points is used. This limits the width of the system and the spatial frequency content of the transverse dimension as shown later. (iii) The gain and density perturbations to the dielectric constant in (1) are applied as complex gain sheets at discrete intervals up and down the resonator cavity. This approximation may only affect the quantitative accuracy of the results. (iv) Equation (6) is simplified by expanding $q$ in a Taylor series about $x$ and using a summation to approximate the time integration. These approximations are numerically accurate as long as the distance between data points and the time increment in the summation are small.

\section{NUMERICAL PROCEDURES}

\section{A. Free-Space Propagation}

In this work, a Fast Fourier Transform (FFT) [20] technique is used to repeatedly solve the freespace wave equation up and down the resonator cavity. The medium effects are applied as complex gain sheets at discrete intervals throughout the cavity. For simplicity, the calculation is limited to one transverse dimension. All physical constants are given in their normal three-dimensional units. The total output power is given in units of power per unit length in the other dimension. Any explicit time dependencies are handled by replacing $t$ with

$$
t=t_{\bar{n}}=\bar{n} \times\left(\frac{2 L}{c}\right)
$$

where $\bar{n}$ is the number of trips around the resonator. To begin, equation (9) is rewritten in a dimensionless form for a fixed time interval, $t_{\bar{n}}$,

$$
\left[\frac{\partial^{2}}{\partial x^{2}}-4 \pi i N_{f} \frac{\partial}{\partial z}\right] u\left(x, z, t_{\bar{n}}\right)=-2 \pi i N_{f} G_{c}\left(x, z, t_{\bar{n}}\right) u\left(x, z, t_{n}\right)
$$


where $x$ is scaled by the transverse radius, $a$, of the output mirror, and $z$ is scaled by the length, $L$, of the cavity. The Fresnel number, $N_{f}$, is defined by

$$
N_{f} \equiv \frac{a^{2}}{L \lambda}
$$

and the envelope, $\tilde{E}$, of the electric field is normalized according to

$$
|u|^{2} \equiv \frac{I}{I_{s a t}}=\frac{\frac{1}{2} n c|\tilde{E}|^{2}}{I_{s a t}}
$$

Here the dielectric constant of (1), which contains all of the medium effects, is rewritten as a complex gain profile,

$$
G_{c}\left(x, z, t_{\bar{n}}\right) \equiv-i k L \Delta \epsilon\left(x, z, t_{\bar{n}}\right)=g L-4 \pi i k L a\left(\frac{\rho}{m_{0}}\right)
$$

where the real part is the gain and the imaginary part is the phase shift due to the density variation.

In order to solve (11), the standard gain sheet approximation [18] is assumed by dividing the cavity into $N_{s}$ sections, as depicted in Figure 2, and free-space propagation through each of these sections is assumed. All of the medium effects accumulated by the beam as it passes through a section are lumped together into a single, transverse complex gain profile (sheet), which is applied to the beam after freespace propagation through a section. To accomplish this approximation, $G_{c}$ is assumed to vary slowly along the axis of the resonator by making the following substitution:

$$
u\left(x, z, t_{\bar{n}}\right)=U\left(x, z, t_{\bar{n}}\right) \exp \left(\frac{G_{c} z}{2}\right)
$$

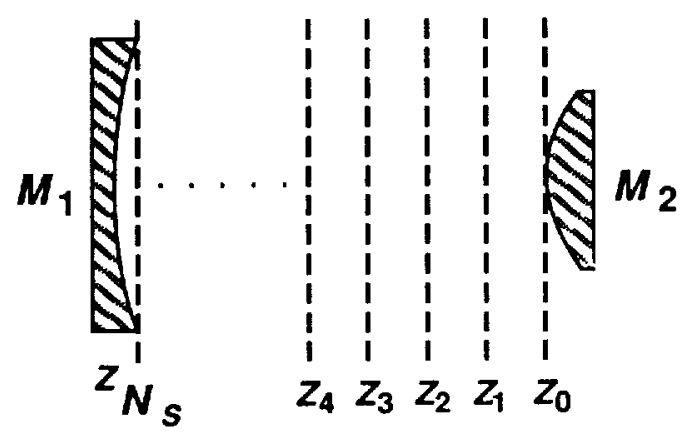

Figure 2. Resonator divided into $N_{s}$ sections. 
Equation (11) now takes the form of a free-space wave equation,

$$
\left[\frac{\partial^{2}}{\partial x^{2}}-4 \pi i N_{f} \frac{\partial}{\partial z}\right] U\left(x, z, t_{\bar{n}}\right)=0
$$

Taking the FFT, equation (17) is obtained

$$
\frac{\partial \tilde{U}\left(\kappa, z, t_{\bar{n}}\right)}{\partial z}=\frac{-i \kappa^{2}}{4 \pi N_{f}} \tilde{U}\left(\kappa, z, t_{\bar{n}}\right)
$$

where

$$
\bar{U}\left(\kappa, z, t_{\bar{n}}\right)=\mathrm{FFT}\left[U\left(x, z, t_{\bar{n}}\right)\right] .
$$

Equation (17) has the solution

$$
\bar{U}\left(\kappa, z, t_{n}\right)=\tilde{U}\left(\kappa, z_{0}, t_{\bar{n}}\right) \exp \left[\frac{-i \kappa^{2}}{4 \pi N_{f}}\left(z-z_{0}\right)\right]
$$

Thus, the propagation of a wave front through a section is given by

$$
U\left(x, z_{m}, t_{\bar{n}}\right)=\mathrm{FFT}^{-1}\left[\exp \left(\frac{-i \mathrm{~K}^{2}}{4 \pi N_{f}} \Delta z\right) \mathrm{FFT}\left[U\left(x, z_{m-1}, t_{\bar{n}}\right)\right]\right],
$$

where $z_{m-1}$ and $z_{m}$ are the longitudinal positions of the front and back edges of the $m^{\text {th }}$ section, and

$$
\Delta z=z_{m}-z_{m-1}=\frac{1}{N_{s}} .
$$

Remember that the $z$-dimension has been normalized to the length of the cavity. 


\section{B. Complex Gain Profile}

After free-space propagation through a section, the medium effects are applied as a complex gain sheet which, according to (15), yields

$$
u\left(x, z_{m}, t_{\bar{n}}\right)=\exp \left(\frac{1}{2} G_{c}\left(x, z_{m}, t_{\bar{\pi}}\right) \Delta z\right) \times \mathrm{FFT}^{-1}\left[\exp \left(\frac{i \mathrm{~K}^{2}}{4 \pi N_{f}} \Delta z\right) \operatorname{FFT}\left[u\left(x, z_{m-1}, t_{\bar{n}}\right)\right]\right] .
$$

The use of complex gain sheets is valid as long as $G_{c}\left(x, z_{m}, t_{\bar{n}}\right)$ varies slowly over the distance through a section. Since $G_{c}$ contains the medium effects, its size should match that of the lasing medium inside the laser cavity. In our calculations, it is assumed that the medium fills the entire cavity and $G_{c}$ covers the entire calculation space. The time dependence of $G_{c}$ is accommodated by using the field profile, $u\left(t_{\bar{n}-1}\right)$, of the previous trip to calculate a new complex gain profile, $G_{c}\left(t_{\bar{n}}\right)$, for the next trip. In essence,

$$
G_{c}\left(t_{\bar{n}}\right)=f\left[u\left(t_{\bar{n}-1}\right)\right]
$$

Now $G_{c}$ is broken down into its real and imaginary parts,

$$
G_{i}=G_{r}+i G_{i}
$$

and each part is calculated independently. According to (14) and (2), the real part of the complex gain, $G_{r}$, depends explicitly on the intensity and is given by

$$
G_{r}\left(x, z_{m}, t_{\bar{n}}\right)=\frac{g_{0}}{1+\left|u^{+}\left(x, z_{m}, t_{n}\right)\right|^{2}+\left|u\left(x, z_{m}, t_{\bar{n}}\right)\right|^{2}}
$$

where the + and - superscripts denote the forward- and backward-traveling wave.

Calculation of the imaginary part, $G_{i}$, of the complex gain profile is not so straightforward since it involves solving the time integral of (6). Equation (6) is substituted into (14) to obtain

$$
\begin{aligned}
G_{i}\left(x, z_{m}, t_{\bar{n}}\right) & =\left(\frac{4 \pi k L a(\gamma-1)}{m_{0} C_{s}^{2}}\right) \times \int_{0}^{t_{\bar{n}}} d t^{\prime}\left[q\left(x, z_{m}, t^{\prime}\right)-\frac{1}{2} q\left(x+C_{s}\left(t_{\bar{n}}-t^{\prime}\right), z_{m}, t^{\prime}\right)\right. \\
& \left.-\frac{1}{2} q\left(x-C_{s}\left(t_{\pi}-t^{\prime}\right), z_{m}, t^{\prime}\right)\right]
\end{aligned}
$$


The integral is evaluated by expanding $q$ in a Taylor series about $x$, substituting a summation for the integration, and transforming the summation into a form that can be computed with a running total, rather than performing the summation every time. The second derivative of $q$ with respect to $x$ resulting from the Taylor series expansion is computed with finite-difference differentiation formulas [21]. As mentioned before, the accuracy of these approximations is good as long as the distance between data points and the time increment in the summation are small. Appropriate increments are determined by repeatedly performing the calculations with smaller and smaller increments, until the desired accuracy is obtained. In our calculations, $N_{s}=10$ complex gain sheets, 256 transverse data points, and a time increment equal to the round-trip propagation time of light are used.

\section{Feedback and Output Mirrors}

After the EM wave has been numerically propagated from one end of the resonator to the other, it is necessary to model the reflection of the wave from the end mirror. For the hard-edge unstable resonator, it is assumed that the end mirrors are perfectly reflecting and spherical. Reflection from a spherical mirror induces a phase shift in the wave front. This phase shift is given by [22]

$$
\phi=-2 k\left[R_{m}-\sqrt{R_{m}^{2}-x^{2}}\right]=\frac{-k x^{2}}{R_{m}}\left[1+\frac{x^{2}}{4 R_{m}^{2}}+\ldots\right],
$$

where $R_{m}$ is the radius of curvature of the mirror, and $\phi$ has been expanded with a Maclaurin series in $x$. $R_{m}$ is taken to be positive for a concave mirror and negative for a convex mirror. In the paraxial limit $\left(x^{2} / 4 R_{m}<<1\right)$, reflection from the feedback mirror (mirror 1$)$ is given by

$$
u^{+}(x, 1, \bar{n})=u^{-}(x, 1, \bar{n}) \exp \left(\frac{-i k x^{2}}{R_{1}}\right)
$$

For the output mirror (mirror 2), a similar expression with only a minor modification to account for the output coupling of the laser is used. Because the output is taken outside the edge of the output mirror, there is no reflected wave beyond this edge. Therefore, reflection from the output mirror is given by

$$
u^{+}(x, 0, \bar{n}+1)= \begin{cases}u^{-}(x, 0, \bar{n}) \exp \left(\frac{-i k x^{2}}{R_{2}}\right) & |x|^{2}<a \\ 0 & |x|^{2}>a\end{cases}
$$

where $a$ is the transverse radius of the output mirror. Diffraction is neglected beyond the edge of the feedback mirror because it is small in comparison with the output coupling of the laser. 
Replacing the hard-edge output mirror with a Gaussian-reflectivity mirror (GRM) is accomplished by replacing (29) with

$$
u^{+}(x, 0, \bar{n}+1)=u^{-}(x, 0, \bar{n}) \sqrt{\tilde{R}(x)} \exp \left(\frac{-i k x^{2}}{R_{2}}\right)
$$

where the reflectivity, $\tilde{R}(x)$, of the GRM is given by [16]

$$
\tilde{R}(x)=\tilde{R}_{0} \exp \left(\frac{-2 x^{2}}{a^{2}}\right),
$$

where $a$ is the $1 / e$ of the GRM and $\tilde{R}_{0}$ is the reflectivity at the center $(x=0)$.

\section{Numerical Problems}

Several numerical problems, including aliasing, energy leakage, and Gibbs" phenomenon. must be accounted for when using the FFT to model wavefront propagation. These problems have heen addressed in a previous work [18]. For the sake of completeness, aliasing and energy leakage are briefly discussed. Gibbs" phenomenon is discussed more extensively because of an associated problem not noted in Reference 18.

Aliasing occurs when the number of data points is too few for the FFT to adequately resolve the frequency content of the wavefront. For the hard-edge resonator, aliasing limits the use of the FFT to small Fresnel numbers, since the spatial frequency content of the wavefront is proportional to the Fresnel number. For large Fresnel numbers, the number of data points needed to sample the high frequency content of the wavefront becomes prohibitive. By trial and error, it is determined that 256 data points are adequate for the small Fresnel number results presented here.

Next, the energy leakage problem is addressed. Unlike the continuous Fourier transform, whose integration limits are infinite, the FFT's integration limits are finite. Thus, the FFT actually solves the problem of a cyclic function, where the sampled function, in this case the wavefront inside the resonator, represents one complete cycle of the cyclic function. The guard band separating the apertures of each cycle must be large enough to prevent diffracted energy from leaking into adjacent cycles as the solution is propagated down the resonator. Assuming a uniform plane wave illuminating an aperture of width 2a and using Huygens' integral to estimate the fractional energy falling outside a band of $-G_{b} a \leqslant x \leqslant G_{b} a$, Sziklas and Siegman [18] developed a criterion for the guard band ratio which is given by

$$
G_{b} \geqslant 1+\frac{1}{2 \pi^{2} N_{c} \epsilon b}
$$


where $\epsilon_{b}$, is the fractional energy spill-over that can be tolerated, and $N_{c}$ is the collimated Fresnel number which is given by

$$
N_{c}=\frac{M^{2}}{M+1}\left(\frac{a^{2}}{l \lambda}\right)=\frac{M^{2}}{M+1} N=\frac{2 M^{2}}{M^{2}-1} N_{q q}
$$

In theory, equation (32) gives a reasonable estimate for $G_{b}$. However, in practice, $G_{b}$ must be determined by trial and error. If $G_{b}$ is too small, then energy leaking from adjacent cells will produce undesirable modulations in the wavefront. Table 1 compares values of $G_{b}$ obtained from (32) with values obtained by trial and error as a function of $N_{e q}$. Here, a fractional energy leakage of $\epsilon_{b}=0.004$ and a magnification of $M=1.4$ are assumed. In practice, $G_{b}$ needs to be larger than the minimum value obtained from (32).

Finally, Gibbs' phenomenon [23] is addressed. When a discretely sampled wavefront, having a sharp cut-off due to a hard-edge aperture, is transformed with the FFT and then back-transformed with the inverse FFT, the original wavefront is reproduced exactly. However, if the transformed wavefront is propagated a short distance and then back-transformed, a numerical inaccuracy, known as Gibbs' phenomenon, is introduced. When a apertured function is transformed, there will be high frequency components due to the sharp edges of the function. Since the number of data points must be finite, the FFT effectively truncates the high frequency components at the highest frequency of the transform. In essence, the FFT acts as a square low-pass frequency filter. Thus, when the transformed wavefront is propagated a short distance, some inaccuracy will be introduced. The iterative application of the FFT, propagation, and inverse FFT to perform the mode calculation compounds the problem.

To reduce or eliminate Gibbs' phenomenon, it is necessary to replace the square, low-pass filter with a filter that has a more gradual cut-off frequency. There are many different filters that can be used for this purpose [23]. This work employs a raised cosine filter [18], also known as a Hanning filter [23], depicted in Figure 3(a). By experimenting with several variations of this filter, it was determined that the rounded corners of the filter are primarily responsible for the correction of Gibbs' phenomenon. For the hard-edge resonator calculations performed here, it was found that the truncated filter of Figure 3(b) yields an accurate solution with the fewest data points. Another filter which may be even better is an extended cosine-bell filter [25], where a raised cosine filter is applied to the first and last 10 percent of the data and a weight of unity is applied between. All of the hard-edge resonator calculations are performed with the truncated Hanning filter of Figure 3(b).

The correction of Gibbs' phenomenon introduces another problem which has not been addressed before. The application of the frequency filter introduces an artificial power loss which is compounded by the fact that the filter is applied several times up and down the cavity; twice for each complex gain sheet in the cavity. In a previous work [18], the artificial power loss was not significant since only one gain sheet was used. However, in this work 10 gain sheets are used, and the filter is applied 20 times. The power loss does not affect the qualitative significance of the results, but a comparison for different Fresnel numbers becomes meaningless. When the Fresnel number of the hard-edge resonator is increased, the mode volume increases and, as a consequence, the total output power should also increase. However. the frequency of the modulations in the mode increases at the same time, resulting in an increased power loss due to the frequency filter. At smaller Fresnel numbers, the increasing mode 
TABLE 1. COMPARISON OF VALUES OF $G_{b}$, CALCULATED FROM EQUATION (32) AND OBTAINED BY TRIAL AND ERROR

\begin{tabular}{|c|c|c|}
\hline $\mathrm{N}_{\mathrm{cq}}$ & Calculated & Trial and Error \\
\hline 1 & 4.0 & 4.0 \\
2 & 2.5 & 3.0 \\
3 & 2.0 & 2.5 \\
4 & 1.8 & 2.3 \\
5 & 1.6 & 2.0 \\
\hline
\end{tabular}

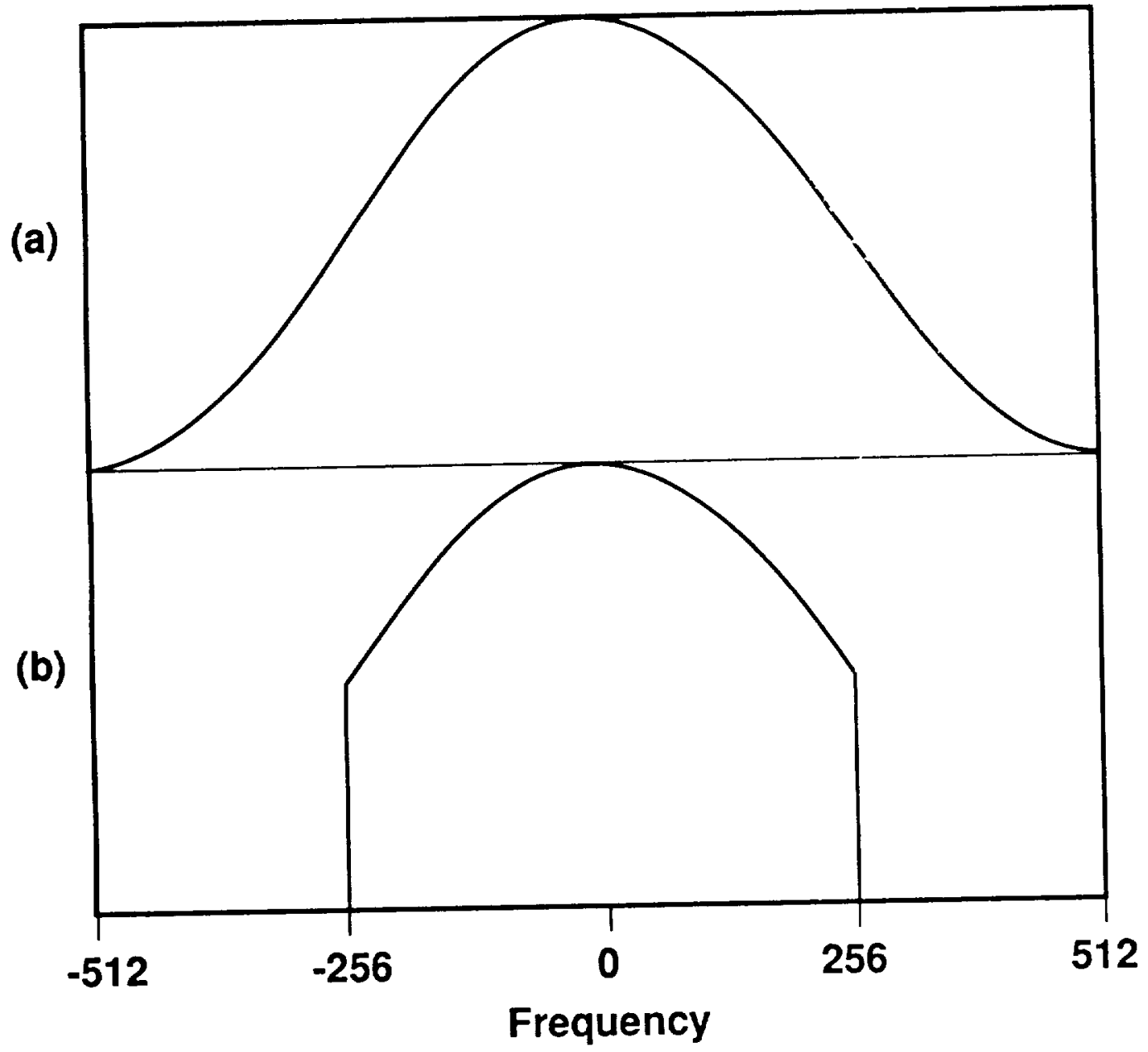

Figure 3. Variations of the Hanning filter: (a) normal and (b) truncated. The maximum frequency of the FFT is proportional to the number of data points. 
volume wins out over the increasing filter losses and the power. output increases. At larger Fresnel numbers, the filter losses win out and the power output decreases. Thus, the quantitative inaccuracies are greater at larger Fresnel numbers.

The following is done to correct the artificial power loss: (1) determine the percentage of energy removed by the filter, and (2) evenly distribute the loss back into the wavefront. This procedure turns out to be important for quantitative comparisons [26].

\section{RESULTS}

The numerical model developed in the previous section is used to model the time evolution of the mode of the unstable resonator; first with a conventional hard-edge output mirror, and then with a corrective Gaussian-edge output mirror. Figure 4 presents the time history of the intensity and phase profile just before the output mirror for the hard-edge resonator. At $2 \mu$ s the heating has very little effect on the mode of the resonator, and the plots of Figure $4($ a) are representative, in shape, of the mode of the equivalent passive resonator. As the heating builds, the number of diffraction ripples in the intensity profile double. and the intensity becomes more strongly peaked near the axis of the resonator compared with the intensity at the edge of the output mirror $(x=1)$. In addition, the peak-to-peak swing of the phase profile grows from about $40^{\circ}$ at $2 \mu$ s to about $145^{\circ}$ at $18 \mu \mathrm{s}$. Aiter $20 \mu$ s the mode becomes very unstable and no useful cavity oscillation is obtained.

A quantitative comparison is begun by varying the equivalent Fresnel number, $N_{\text {eq }}[24]$, which is an important parameter in determining the mode of a hard-edge unstable resonator and is roughly equal to the number of modulations in half of the intensity profile. The dependence of the $\mathrm{MMI}$ on $N_{\text {cat }}$ is somewhat complicated because the structure of the resonator mode is determined by diffraction from the edge of the output mirror. As $N_{\text {eq }}$ is increased, the number of modulations in the mode increase. Thus, the MMI effects are expected to increase as well. However, there is also a corresponding decrease in the amplitude of the modulations which may compensate for the increased number of modulations. Another factor affecting the dependence of the MMI on $N_{e q}$ is related to the amount of power extracted from the resonator. Due to the highly diffractive nature of the unstable resonator, the round-trip losses of any given mode do not vary consistently with increasing values of $N_{c q}$. It has been demonstrated in previous passive resonator calculations [10.24] that as $N_{c \prime}$ is increased the round-trip losses approach a constant value with a periodic ripple about this value. The peaks of the ripple occur at approximately integer values of $N_{\text {' }}$ where two different modes cross. As one of these peaks is approached, the losses of the current lowest-loss mode are decreasing. At the peak, both modes exhibit the same round-trip losses, and as the peak is passed the mode with decreasing losses becomes the new lowest-loss mode. This periodic dependence of the energy may help to produce a complex dependence of the MMI on $N_{\text {eq }}$. Finally, $N_{\text {'c } q}$ affects the scale of the density variation relative to the size of the mode, which increases with increasing

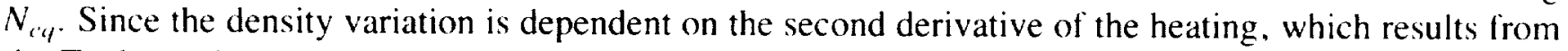
the Taylor series expansion of equation (26), the scale of the density variation is expected to be reduced relative to the size of the mode as $N_{e q}$ is increased. Together, these four factors cause the MMI to display a complex dependence on $N_{\text {c' } q}$.

The complicated dependence of the MMI on $N_{c q}$ is borne out in the plots of beam quality and peak density variation in Figures 5 and 6, respectively. The beam quality is defined as the ratio of the far-field 
Intensity

(a)

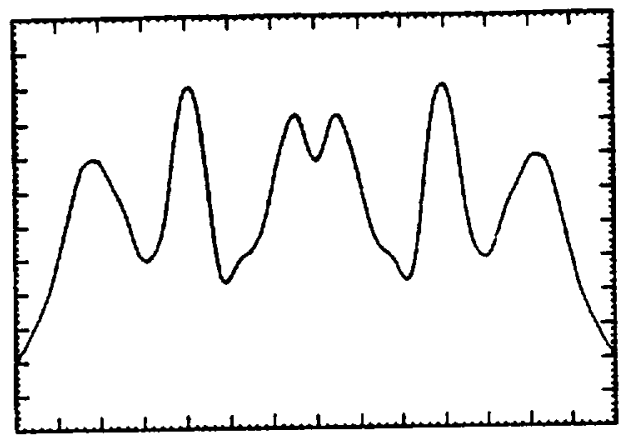

(b)

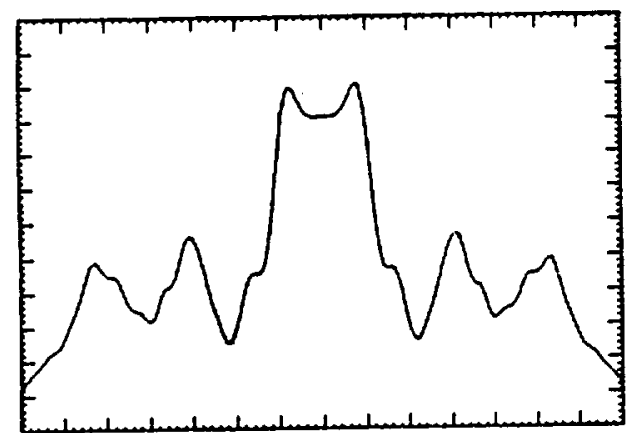

(c)

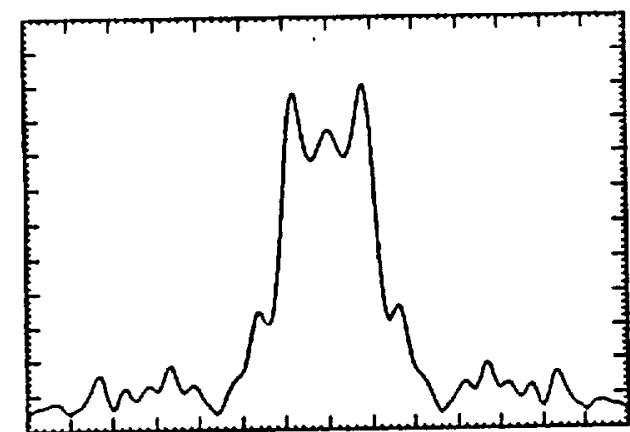

(d)

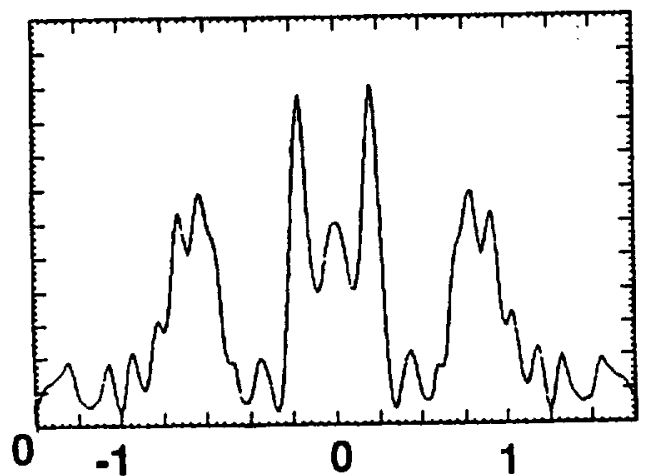

Phase
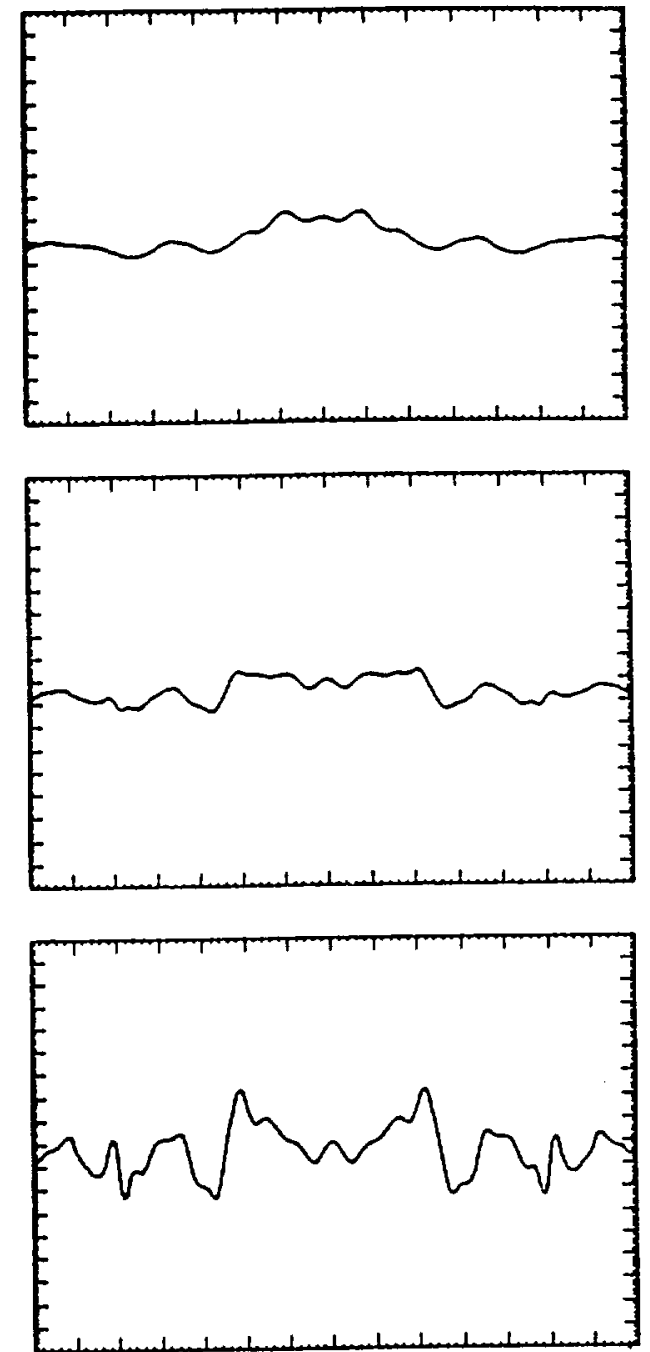

180

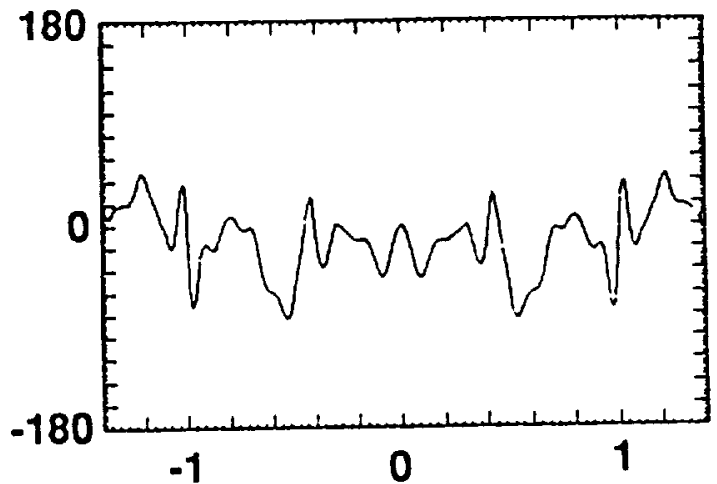

Figure 4. Time evolution of intensity and phase profile just before the hard-edge output mirror with $N_{e q}$ $=2.0$ and $P=0.3 \mathrm{~atm}$. The intensity is normalized to 1 at its peak value, the phase is plotted relative to its value at position $x=0$, and the position is in units of the width of the output mirror. The labels (a), (b), (c), and (d) refer to the intensity and phase profile at time $2,8,12$, and $16 \mu \mathrm{s}$, respectively. 


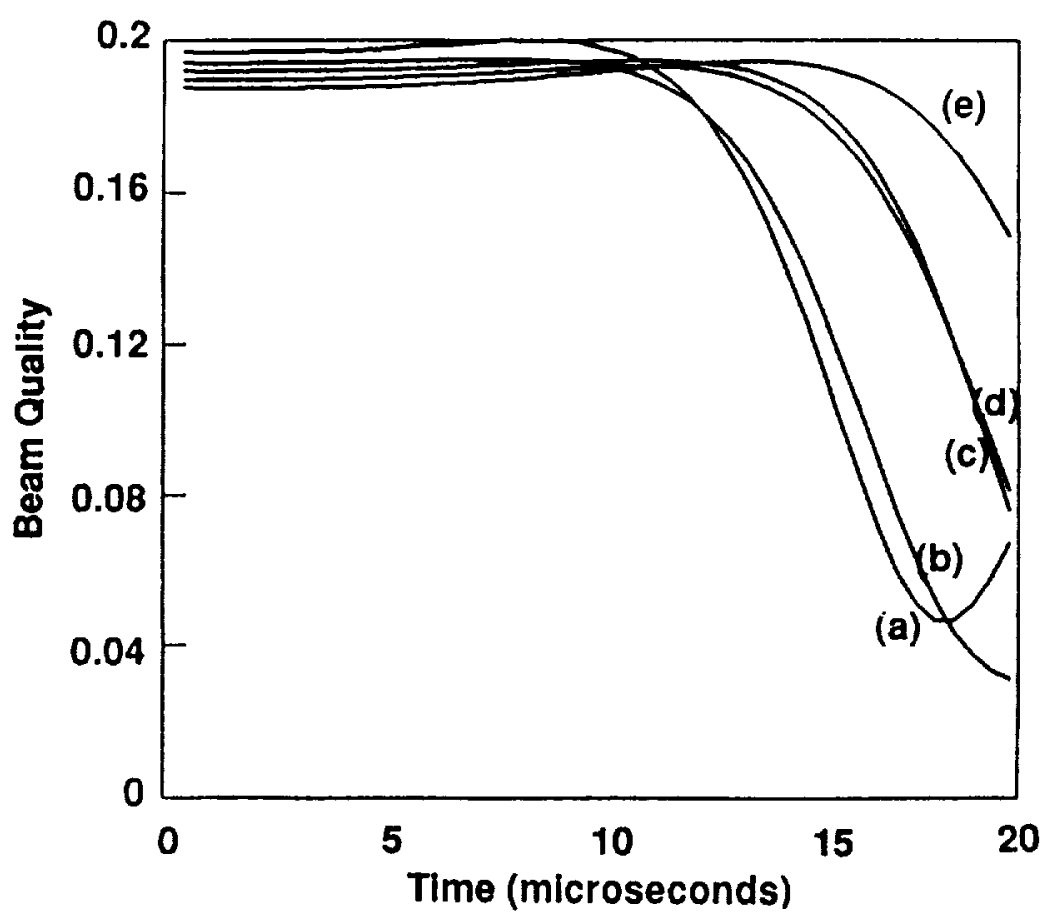

Figure 5. Hard-edge resonator beam quality as a function of time with $P=0.3 \mathrm{~atm}$ and $N_{\text {eq }}=$ (a) 1.0 . (b) 2.0, (c) 3.0 , (d) 4.0, and (e) 5.0.

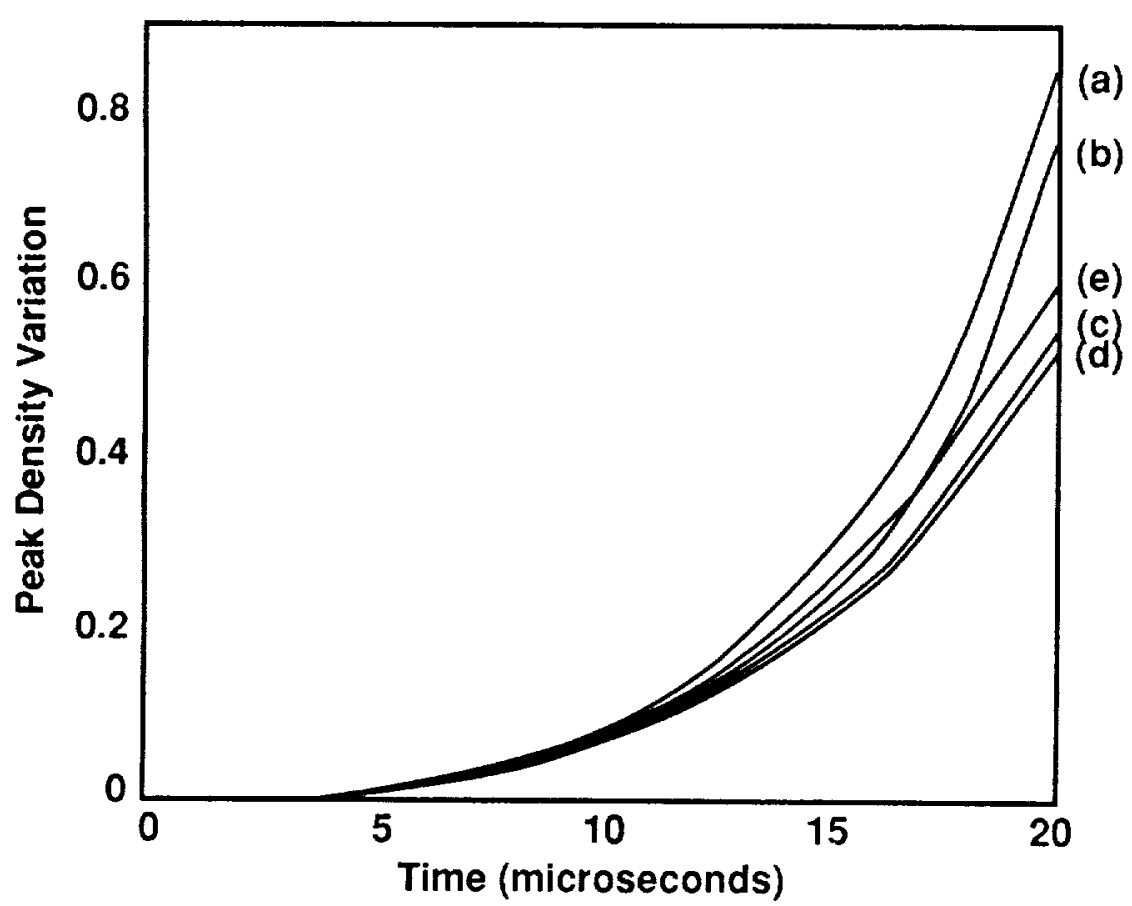

Figure 6. Peak value of the $G_{i}$ profile just before the hard-edge output mirror and as a function of time with $P=0.3 \mathrm{~atm}$ and $N_{t^{\prime} \prime}=$ (a) 1.0 , (b) 2.0 , (c) 3.0 , (d) 4.0 , and (e) $5.0 . G_{i}$ is in units of radians. This plot shows the growth of the density variation. 
power in the central part of the beam to that expected for a diffraction limited Gaussian beam. In other words, the beam quality is a measure of the percentage of total near-field power delivered to the far-field compared with an ideal case. As the heating builds and the beam becomes more unstable, less energy will be delivered to the far-field, and the beam quality will degrade. The density variation is proportional to dimensionless $G_{i}(x)$ in equation (26). For peak density variation, the largest value of $G_{i}(x)$ is used just before the output mirror and as a function of time. This quantity measures the growth of the largest rarefaction or compression region in the medium.

In general, the beam quality in Figure 5 remains fairly constant at about 19 percent, until it begins to degrade in the 10- to $15-\mu$ s range and becomes totally unstable sometime after $20 \mu \mathrm{s}$. The cases of $N_{e q}$ $=1.0$ and 2.0 degrade first, then 3.0 and 4.0, and finally 5.0. The tail of the $N_{e q}=1.0$ plot shows an improvement in beam quality which is not to be trusted since the improvement occurs near the time when the mode of the resonator becomes unstable. The quantitative differences between the plots of beam quality for 1.0 and 2.0, and likewise for 3.0 and 4.0, are small. However, for larger increases in $N_{e q}$ the trend shows an improvement in beam quality. It will be seen later that the initial value of 19 percent is low compared with that of the Gaussian-edge resonator. The important factor that is emphasized here is how quickly the beam quality degrades relative to the onset of lasing. The initial beam quality can be improved considerably by increasing the magnification [24] of the unstable resonator. The plots of peak density variation in Figure 6 show a more consistent behavior. Except for the case of $N_{e q}=5.0$, where the small $N_{e q}$ approximation breaks down, with each increase in $N_{e q}$ the peak density variation decreases which indicates a decrease in the rate of growth of the largest compression or rarefaction region in the cavity.

Now, the pressure of the gas is varied inside the cavity. Since the saturation intensity is proportional to the square of the pressure [26], the amount of heating and the MMI should be reduced when the pressure inside the cavity is reduced. The plots of beam quality and peak density variation in Figures 7 and 8 bear this out. This solution to the MMI is not acceptable however, since the reduced saturation intensity, according to equation (2), causes the gain to saturate at a lower cavity intensity, thus reducing the peak output power.

In order to eliminate the diffraction from the edges of the output mirror, the hard-edge output mirror is replaced with a Gaussian-edge mirror. The time history of the intensity and phase profile obtained from a typical Gaussian-edge resonator calculation is presented in Figure 9 . The plot at $2 \mu s$ is representative of the mode of the equivalent passive resonator, and, as expected, has a Gaussian shape with a flat phase profile which is characteristic of a parallel beam. The initially smooth intensity profile should produce a more evenly distributed heating rate. As the heating builds, the intensity profile broadens with a flatter top which eventually develops into a sizable dip, and the phase profile develops a larger and larger curvature which results in a change in focus of the output beam. Eventually, after $50 \mu s$, the mode of the resonator becomes completely unstable.

It is noted here that variations in pressure produce results very similar to those of the hard-edge resonator. For the Gaussian-edge resonator, only the Fresnel number dependence of the MMI will be examined. Although $N_{e q}$ is no longer important in determining the mode of the resonator, for simplicity it will continue to be used. The Fresnel number dependence is much less complicated for the Gaussianedge resonator, because the relative shape of the mode does not change with changes in the Fresnel number. Only the size of the mode increases with increasing Fresnel number. As mentioned earlier, the density variation is dependent on the second derivative of the heating. Thus, with increasing Fresnel 


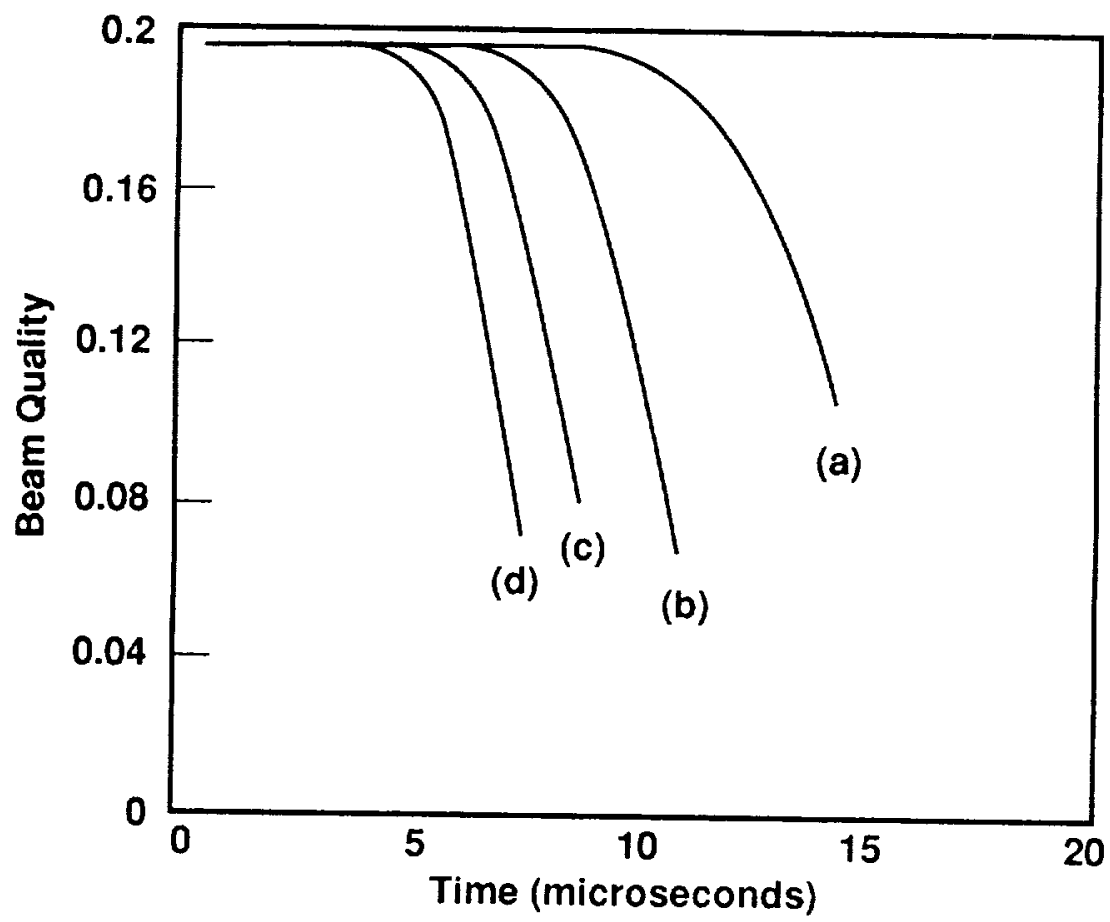

Figure 7. Hard-edge resonator beam quality as a function of time with $N_{e q}=2.0$ and $P=$ (a) 0.3 , (b) 0.5 . (c) 0.7 , and (d) $0.9 \mathrm{~atm}$.

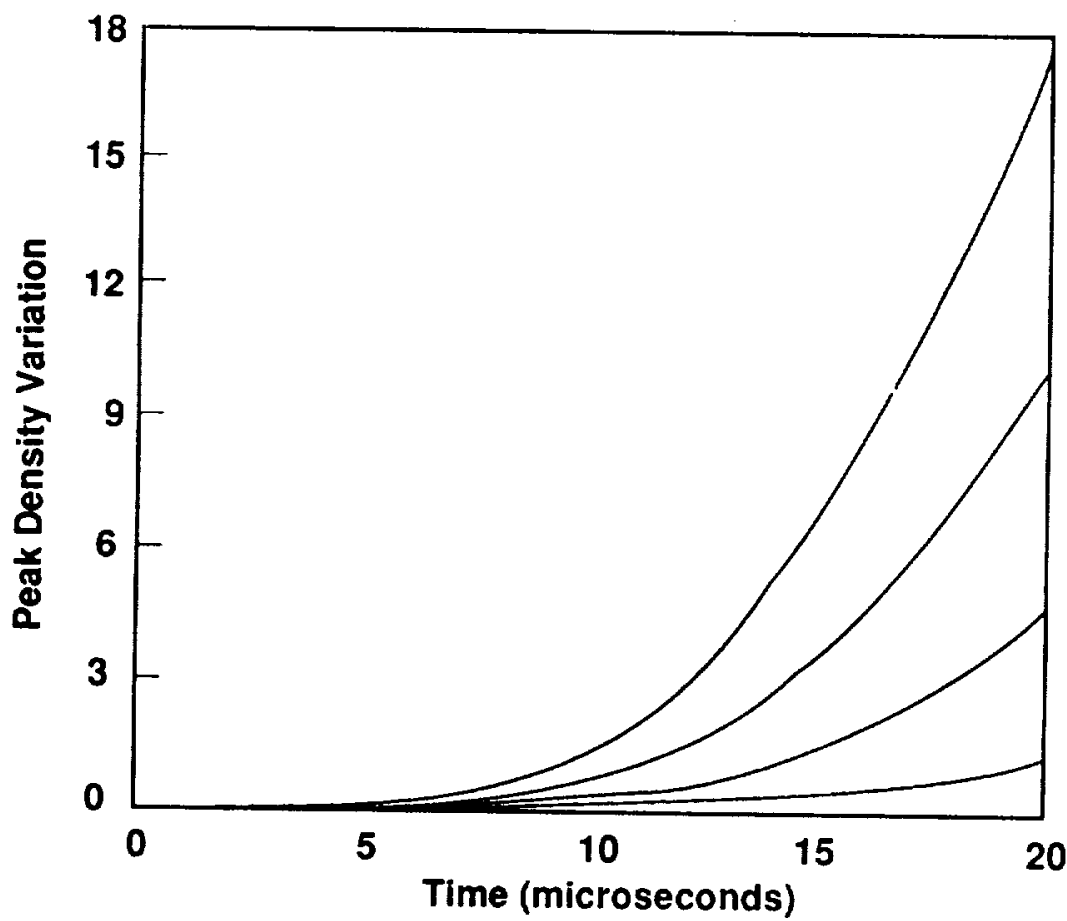

(d)

(c)

(b)

(a)

Figure 8. Peak value of the $G_{i}$ profile just before the hard-edge output mirror and as a function of time with $N_{e^{\prime} q}=2.0$ and $P=$ (a) 0.3 , (b) 0.5 , (c) 0.7 , and (d) 0.9 atm. $G_{i}$ is in units of radians. This plot shows the growth of the density variation. 

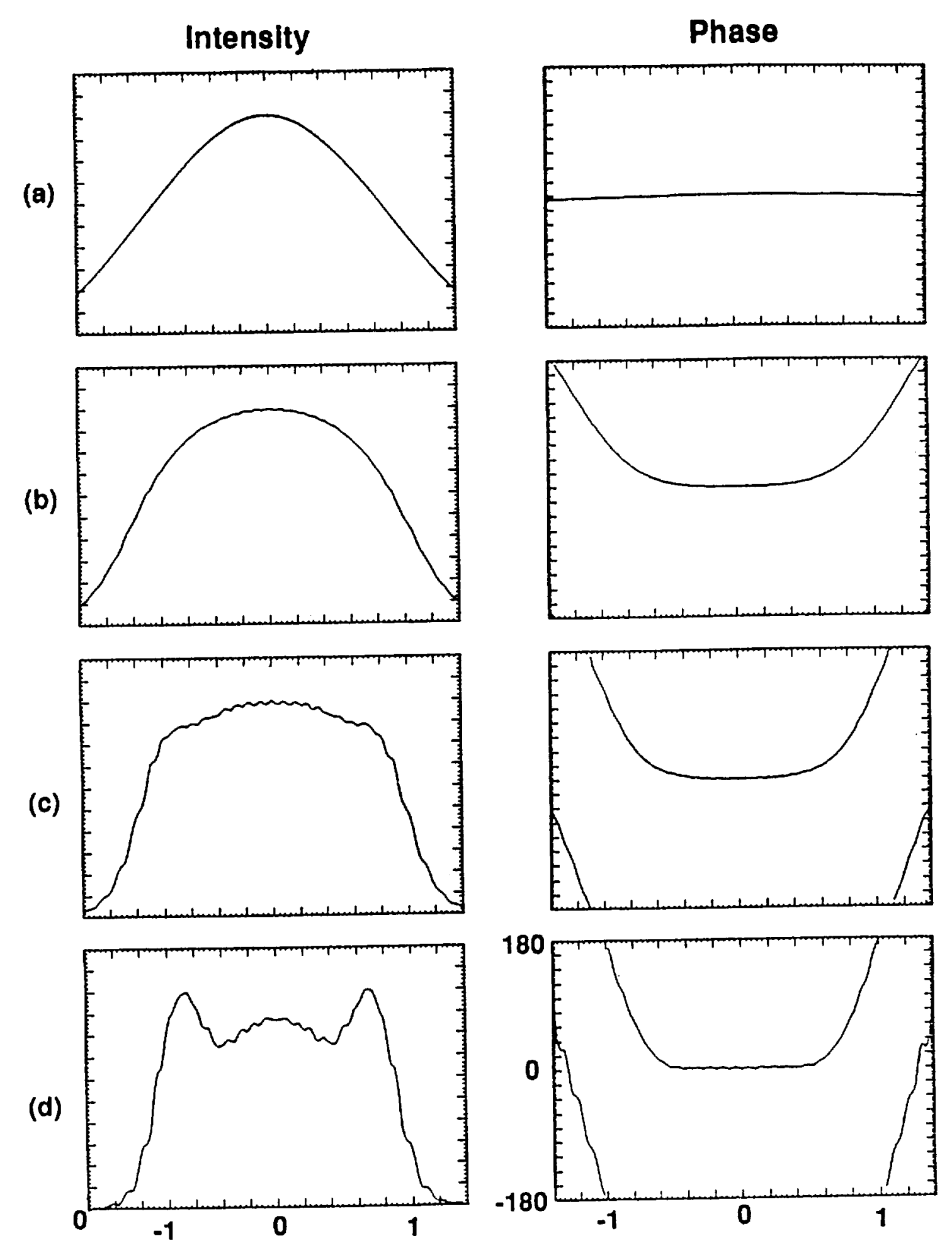

Figure 9. Time evolution of intensity and phase profile just before the soft-edge output mirror with $N_{c^{\prime} \text { q }}$ $=2.0$ and $P=0.3 \mathrm{~atm}$. The intensity is normalized to 1 at its peak value, the phase is plotted relative to its value at position $x=0$, and the position is in units of the width of the output mirror. The labels (a), (b), (c), and (d) refer to the intensity and phase profile at time 2, 40, 46. ind 50 () 4 . respectively. 
numbers, the scale of the heating should be reduced relative to the size of the mode. Consequently, for larger Fresnel numbers a reduction is expected in the MMI effects, which is borne out in the plots of beam quality and peak density variation in Figures 10 and 11. With each increase in Fresnel number, the beam quality improves and the peak density variation grows more slowly. These trends are more obvious than the previous hard-edge resonator cases.

In summary, the beam quality is much better than that of the hard-edge resonator. The initial value is near 100 percent compared with 19 percent for the hard-edge resonator. As mentioned before, the initial value is not important, since the initial beam quality can be improved by increasing the magnification [24] of the hard-edge unstable resonator. More importantly, the beam quality remains fairly constant for a longer period of time than the hard-edge resonator. The beam quality begins to degrade in the 20 - to $30-\mu$ s range compared with the 10 - to $15-\mu$ s range of the hard-edge resonator.

\section{CONCLUSION}

A three-level lasing medium in general and $\mathrm{CO}_{2}$ in an unstable resonator in particular have been modeled. The mode-medium instability is explored numerically for various parameters in the small equivalent Fresnel number $N_{c q}$ regime. The results are in agreement with those of the large Fresnel number regime [9], namely, the beam quality is not too sensitive to $N_{e q}$, although the larger values of $N_{e^{\prime} q}$ seem to provide a better beam quality for practical consideration. A lower pressure or density of the medium certainly improves the beam and prolongs the duration of a high quality beam at the expense of peak power.

In contrast to the case of large $N_{e q}$, the numerical calculations here are rather difficult, and a careful review of various potential problems for numerical inaccuracy are given; for example, aliasing, energy leakage, and Gibbs' phenomenon.

The major contribution of the paper is to show that the soft-edge (Gaussian reflectivity) mirror can indeed improve the beam quality by eliminating the hard-edge mirror diffraction ripples. The useful duration of the beam as well as the quality are much improved. This point is clearly demonstrated in the work. which also turns out to be easier to execute with better accuracy in this case. 


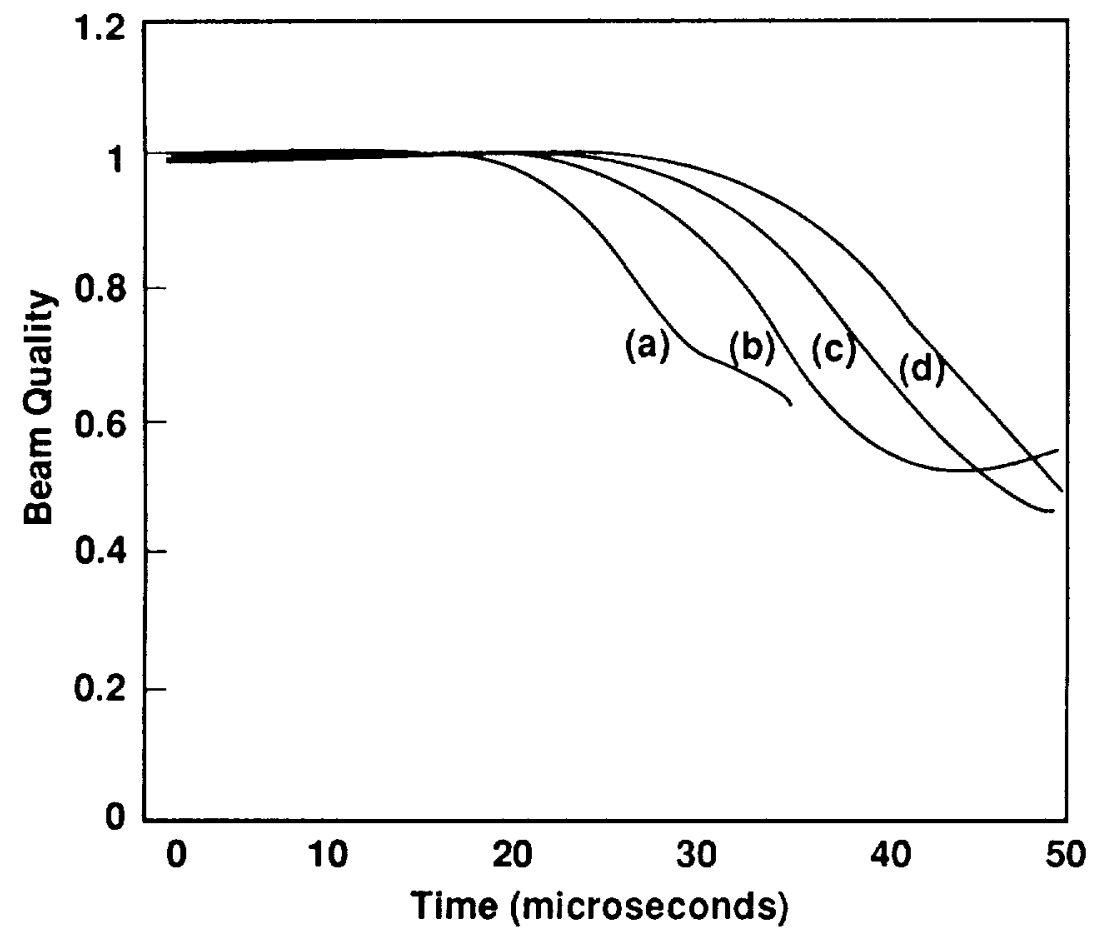

Figure 10. Soft-edge resonator beam quality as a function of time with $P=0.3 \mathrm{~atm}$ and $N_{e q}=($ a) 1.0 , (b) 2.0 . (c) 3.0 , and (d) 4.0 .

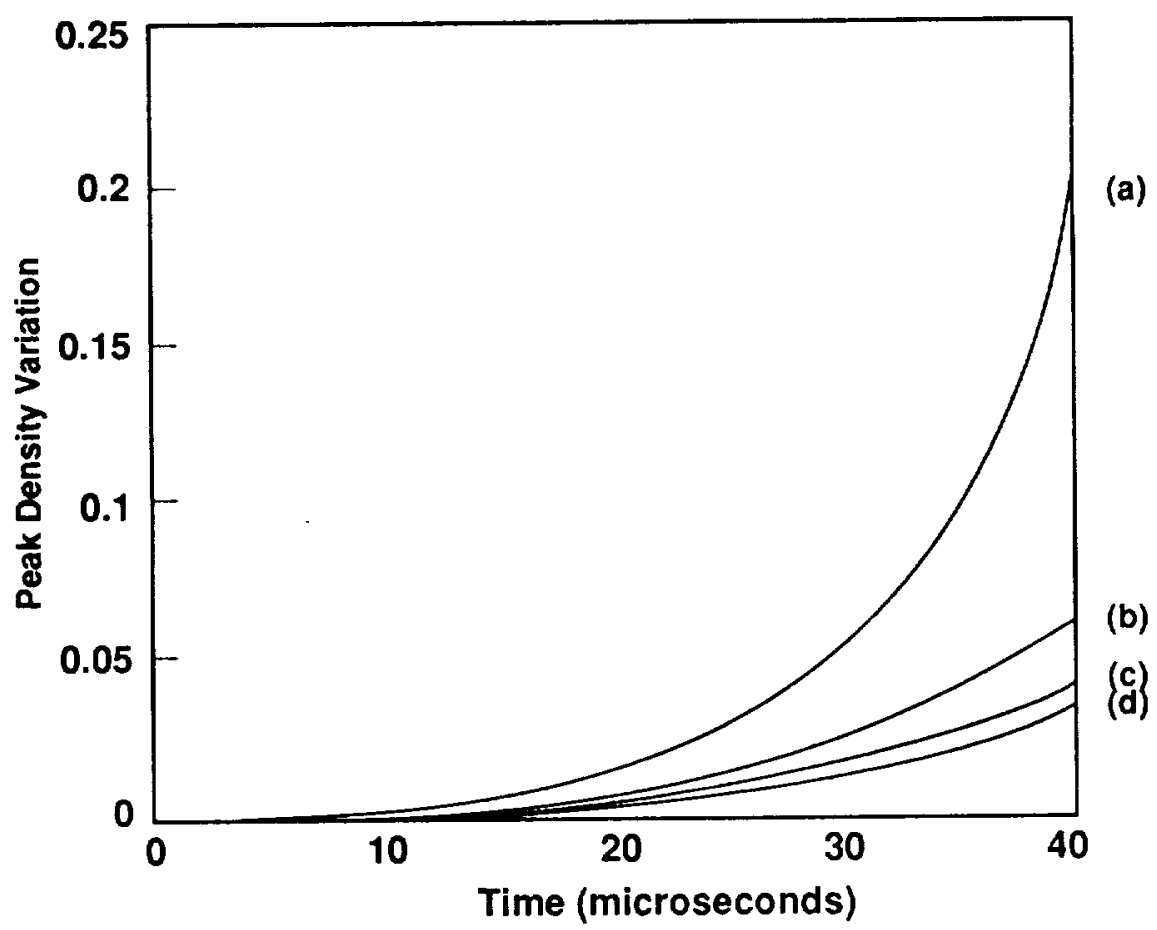

Figure 11. Peak value of the $G_{i}$ profile just before the soft-edge output mirror and as a function of time with $P=0.3 \mathrm{~atm}$ and $N_{c^{\prime} q}=$ (a) 1.0 . (b) 2.0 , (c) 3.0 , and (d) 4.0 . $G_{i}$ is in units of radians. This plot shows the growth of the density variation. 


\section{REFERENCES}

1. Roper, V.G., Lamberton, H.M., and Manley, A.W.J.: "Laser Induced Medium Perturbation in a Pulsed $\mathrm{CO}_{2}$ Laser." Opt. Comm., Vol. 25, 1978, pp. 235-240.

2. Gorton, E.K., Gorton, P.J., and Parcell, E.W.: "LIMP in Continuously Coupled Unstable Resonators." J. Phys. D, Vol. 16, 1983, pp. 517-524.

3. Gorton, E.K., and Parcell, E.W.: "Thermal Defocusing (LIMP) in Stable $\mathrm{CO}_{2}$ Resonators." J. Phys. D, Vol. 16, 1983, pp. 1827-1835.

4. Longaker, P.R., and Litvak, M.M.: "Perturbation of the Refractive Index of Absorbing Media By a Pulsed Laser Beam.” J. Appl. Phys., Vol. 40, 1969, pp. 4033-4041.

5. Tough, R.J.A., and Willets, D.V.: "Density Perturbations Induced By a Discharge in a Laser Cavity." J. Phys. D, Vol. 15, 1982, pp. 2433-2442.

6. Cronburg, T., Flusberg, A., and Korff, D.: "Lost Energy Identification." Avco Everett Research Laboratory, Inc., Report, 1982.

7. Vysloukh, V.A. and Ognev, L.I.: Sov. J. Quan. Elec., Vol. 11, 1981, pp. 727-730.

8. Morris, J., and Stettler, J.: "Electric Discharge Laser (EDL) Mode Media Interaction Theory." Applied Research, Inc., Report No. ARI/R-85-109, 1984.

9. Sung, C.C., Li, Y.Q., and Smithers, M.E.: "Mode-Medium Instability in an Unstable Resonator." Applied Optics, Vol. 27, 1988, pp. 58-65.

10. Fox, A.G., and Li, T.: "Resonant Mode in a Maser Interferometer." Bell Syst. Tech. J., Vol. 40, 1960, pp. $453-484$.

11. Smithers, M.E., Salvi, T.C., and Dente, G.C.: "Unstable Resonator With Canceling Edge Waves." Applied Optics, Vol. 21, 1982, pp. 729-732.

12. Ferguson, T.R., Smithers, M.E., and Perkins, J.F.: "Unstable Resonator With Canceling Edge Waves: Rectangular Aperture." Applied Optics, Vol. 25, 1986, pp. 672-677.

13. McCarthy, N., and Lavigne, P.: "Optical Resonators With Gaussian Reflectivity Mirrors: Misalignment Sensitivity." Applied Optics, Vol. 22, 1983, pp. 2704-2708.

14. Lavigne, P., McCarthy, N., and Demers, J.: "Design and Characterization of Complementary Gaussian Reflectivity Mirrors.” Applied Optics, Vol. 24, 1985, pp. 2581-2586.

15. McCarthy, N., and Lavigne, P.: "Large-size Gaussian Mode in Unstable Resonators Using Gaussian Mirrors." Optics Letters, Vol. 10, 1985, pp. 553-555. 
16. Siegman, A.E.: Lasers. University Science Books, Mill Valley, CA, 1986, pp. 914-919.

17. Willetts, D.V., and Harris, M.R.: "Output Characteristics of a Compact $1 \mathrm{~J}$ Carbon Dioxide Laser With a Gaussian Reflectivity Resonator." IEEE J. Quan. Elec., Vol. 24, 1988, pp. 849-855.

18. Sziklas, E.A., and Siegman, A.E.: "Mode Calculations in Unstable Resonators With Flowing Saturable Gain. 2: Fast Fourier Transform Method." Applied Optics, Vol. 14, 1975, pp. 1874-1889.

19. Svelto, O.: Principles of Lasers. Plenum Press, New York and London, 1982.

20. Brigham, E.O.: The Fast Fourier Transform. Prentice-Hall, Inc., NJ, 1974.

21. Beyer, W.H.: Standard Math Tables. CRC Press, Inc., Boca Raton, FL, 1978.

22. Hecht, E., and Zajac, A.: Optics. Addison-Wesley Publishing Co., Reading, MA, 1974 , pp. $124-125$.

23. Bose, N.K.: Digital Filters Theory and Applications. Elsevier Science Publishing Co., Inc., New York, NY, 1985, pp. 250-271.

24. Siegman, A.E.: Lasers. University Science Books, Mill Valley, CA, 1986, pp. 872-884.

25. Bergland, G.D.: "A Guided Tour of the Fast Fourier Transform." IEEE Spectrum, July, 1960, pp. 41-52.

26. Webster, K.L.: "High Power $\mathrm{CO}_{2}$ Laser Instability and Its Corrections." Doctoral Dissertation, University of Alabama in Huntsville, 1989. 



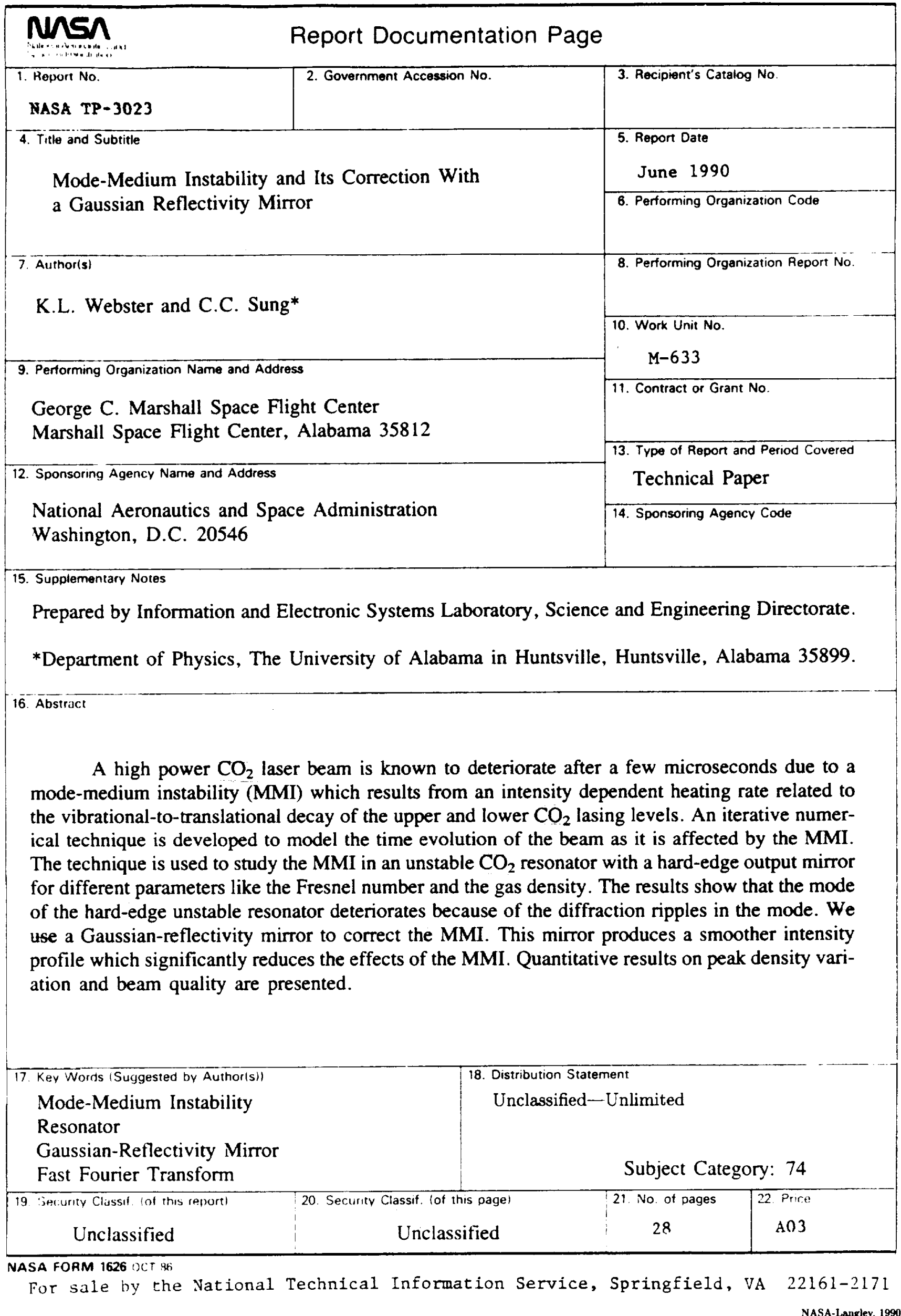


